\title{
Quantitative Beiträge zur Frage des Zusammenwirkens von Ionen und organischen Giften.
}

\author{
II. Mitteilung ${ }^{1}$ ). \\ Von \\ Hans Handovsky. \\ (Aus dem Pharmakologischen Institut der Universität Göttingen.) \\ (Eingegangen am 28. Februar 1922.)
}

In dieser Mitteilung soll die giftverstärkende Wirkung verschiedener Salze auf die Saponinhämolyse der Rz.-Bl. ${ }^{2}$ ) besprochen werden, nachdem in der vorhergehenden einige Gesetzmäßigkeiten im bezüglichen Verhalten des Kochsalzes aufgedeckt wurden.

Zunächst seien im Hinblick auf eine Reihe inzwischen erschienener Arbeiten über das Verhalten von Bl. in vitro einige Bemerkungen zur Methodik vorausgeschickt.

Bei Untersuchungen an $B l$. sind zueierlei Momente zu berücksichtigen:

I.

Aus dem strömenden Blut entfernte Bl. verändern sich ungemein leicht, wie aus eigenen Vorversuchen, besonders aber aus Untersuchungen von Hamburger und seinen Schülern Brinkman, van Creveld, ferner von Ege hervorgeht. So haben Brinkman und van $D^{3} m^{3}$ ) darauf hingewiesen, daß schon die beginnende Gerinnung des Blutes die Bl. so verändert, daß sie Traubenzucker aufnehmen. Hamburger, dann van Creveld ${ }^{4}$ ) haben auf die große Bedeutung des $\mathrm{CO}_{2}$-Gehaltes der Suspensionsflüssigkeit für den Cl-Gehalt der Bl. hingewiesen. van Creveld (a. a. O.) läBt darum das Blut durch paraffinierte Kanülen in paraffinierte Zentrifugierröhrchen unter Paraffinöl einfließen und zentrifugiert sofort schnell; dies dürfte die-schonendste Methode sein; für unsere Versuche waren, wie die Gleichmäßigkeit der Resultate beweist, diese Vorsichtsmaßregeln nicht nötig, zumal wir ja nicht den Elektrolytaustausch, sondern den Endzustand der Veränderung, die Hämolyse, als Indicator benutzten. Der Behauptung von Brinkman und van Dam ${ }^{5}$ ), daß die Blutkörperchen durch Auswaschen mit Salzlösungen ihre Lecithinhülle verlieren sollen, durch Auswaschen mit Rz. nicht, bin ich nicht nachgegangen. Die einzige Vorsichtsmaßregel, auf die ich achtete, war, da $\beta$ die einzelnen Prozeduren nach der Uhr vorgenommen wurden und strenge darauf gesehen wurde, daß sie in allen Versuchen gleich lang dauerten.

\footnotetext{
1) I. Mitteilung, Pflügers Arch. f. d. ges. Physiol. 190, 173.1921.

2) Rz. bedeutet im folgenden Rohrzucker, Bl. rote Blutkörperchen.

$\left.{ }^{3}\right)$ Arch. internat. de physiol. 15, 105. 1919.

$\left.{ }^{4}\right)$ Biochem. Zeitschr. 123, 304. 1921.

5) Biochem. Zeitschr. 108, 35. 1920.
} 
Ich ließ 10-15 ccm Blut aus der Ohrvene von Kaninchen in ein Zentrifugenglas mit Glasperlen einfließen, was stets nur $1-1^{1 / 2}$ Minuten dauerte. Das Blut wurde dann 2 Minuten geschüttelt, koliert, dann je 2,5 ccm in Zentrifugengläser pipettiert, mit Waschflüssigkeit auf ca. $50 \mathrm{ccm}$ aufgefüllt und noch dreimal mit erneuter Waschflüssigkeit je 10 Minuten lang zentrifugiert; hierauf wurde die Waschflüssigkeit abermals abpipettiert, der Bl.-Brei in ein spitzes Zentrifugenglas von ca. $15 \mathrm{ccm}$ Inhalt gespült und 15 Minuten scharf zentrifugiert, dann die Waschflüssigkeit mit einer Capillarpipette möglichst vollständig abgesaugt.

Nun wurde anf zweifache Weise weiter gearbeitet:

Methode I: Die gewaschenen Bl. wurden in den verschiedenen isotonischen Lösungsgemischen aufgeschwemmt, mit ihnen auf $100 \mathrm{ccm}$ aufgefüllt und dann mit 1 Volumprozent verschieden konzentrierter Saponinlösungen versetzt.

Methode II: Die Bl. wurden in doppeltisotonischer Rz.- bzw. Salzlösung aufgeschwemmt, auf $100 \mathrm{ccm}$ aufgefüllt, darauf durch Mischung beider und Verdünnung mit Wasser oder Saponinlösungen unter Vermeidung örtlicher Hämolyse die gewünschten Konzentrationen hergestellt.

Die Aufstellung einer Versuchsserie dauerte 25-30 Minuten, nie länger. Beide Methoden ergaben, wie aus zahlreichen Kontrollversuchen hervorgeht, stets die gleichen Resultate. Meist wurde zur Kontrolle mit beiden Methoden gearbeitet, bei größeren Serien nur mit Methode II. Daß dabei die durch das Pipettieren der Bl.-Suspensionen begangenen Fehler gering waren, erhellt aus folgender $\mathrm{Zu}$ sammenstellung der Versuche in Tab. I. In dieser Tabelle zeigen die Zahlen der ersten Säule an, wie oft in den 53 Versuchen die in der zweiten Säule angegebene Anzahl Bl. in $0,004 \mathrm{cmm}$ gezählt wurden. Die Zählung war stets in der ThomaZeissschen Zählkammer in der üblichen Weise vorgenommen worden.

\section{Tabelle $I$.}

In $0,004 \mathrm{cmm}$ sind enthalten:

\begin{tabular}{|c|c|}
\hline Zahl des Versuche & Erythrocyten \\
\hline 1 & 424 \\
\hline 2 & 425 \\
\hline 4 & 426 \\
\hline 1 & 427 \\
\hline 1 & 428 \\
\hline 8 & 432 \\
\hline 16 & 436 \\
\hline 3 & 438 \\
\hline 8 & 440 \\
\hline 1 & 441 \\
\hline 1 & 442 \\
\hline 3 & 443 \\
\hline 3 & 445 \\
\hline 1 & 446 \\
\hline$\overline{53}$ & Mittel \\
\hline
\end{tabular}

Die Versuchsserien wurden dann in den Brutschrank von $37^{\circ}$ gebracht, darin 2 Stunden belassen, nachdem sich herausgestellt hatte, daß die Hämolyse 2 mal nach 4 Stunden und 2 mal nach 6 Stunden nicht weiter zugenommen hat. Hierauf wurden die Serien mindestens 10 Stunden im Eisschrank belassen und dann der Hämolysegrad mittels Zählung der Bl. bestimmt, nachdem sich aus früheren Untersuchungen ergeben hatte, daß es eine partielle Hämolyse einzelner Bl. in vitro nicht gibt, daß also die Bestimmung der Hämolyse durch Zählung der nicht hämolysierten Bl. und durch colorimetrische Bestimmung des ausgetretenen Hämoglobins 
vollkommen übereinstimmt. Stets wurden die Empfindlichkeiten der in den verschiedenen Suspensionsflüssigkeiten aufgeschwemmten Bl. mit der von Rz.-Bl. derselben Entnahme verglichen; wenn Bl. in Rz. eine abweichende Empfindlichkeit zeigten, was in 61 Versuchen nur $8 \mathrm{mal}$ der Fall war, wurden diese Reihen verworfen. Die gute Übereinstimmung der Hämolysegrade von Bl. verschiedener Entnahme in der gleichen Suspensionsflüssigkeit zeigen die folgenden Tab. II und III.

Tabelle $I I$.

Hämolyse in 7,8 proz. Rohrzucker.

\begin{tabular}{c|cccccccccccc|c}
\hline \hline Saponingehalt & \multicolumn{10}{c|}{ Hämolysegrad $\%$} & Mittel \\
\hline $0,006 \%$ & 14, & 15, & 15, & 15, & 16, & 16. & 16, & 16, & 17, & 17, & 20, & 24 & $16 \%$ \\
$0,008 \%$ & 19, & 20, & 20, & 21, & 23, & 23 & & & & & & $21 \%$ \\
$0,010 \%$ & 22, & 24, & 24, & 25, & 26, & 28, & 28, & 28, & 30 & & & $26 \%$ \\
$0,016 \%$ & 39, & 40, & 41, & 41, & 44 & & & & & & & $41 \%$ \\
$0,025 \%$ & 61, & 62, & 62, & 62 & & & & & $62 \%$
\end{tabular}

Tabelle III.

Hämolyse in 0,9 proz. $\mathrm{NaCl}$.

\begin{tabular}{c|rrrr|r}
\hline \hline Saponingehalt & \multicolumn{3}{|c|}{ Hämolysegrad \% } & Mittel \\
\hline \hline $0,003 \%$ & $6, \quad 8, \quad 8$, & 9 & & $8 \%$ \\
$0,004 \%$ & $55, \quad 56, \quad 59$, & 59, & 62, & 63 & $59 \%$ \\
$0,006 \%$ & $90,90,90$, & & & $90 \%$
\end{tabular}

Auf diese Weise war es möglich, methodische Unzukömmlichkeiten, die beim Arbeiten mit Bl. zu so vielen Fehlschlüssen Anlaß gegeben haben, -- wenigstens für unsere Zwecke - zu vermeiden.

II.

Beim Forschen nach GesetzmäBigkeiten im Verhalten von Bl. stehen wir aber noch einer zweiten Schwierigkeit gegenüber; wir haben es nämlich nicht etwa wie bei chemischen Reaktionen mit vollkommen gleichwertigen Reaktionseinheiten (Molekülen) zu tun, sondern mit notwendigerweise verschieden alten und darum verschieden reaktionsfähigen $\left.^{1}\right)$. Wir wollen diesen Zustand Heterovitalität nennen; die Bl.Suspension ist ein heterovitales System, und zwar sind die jungen $B l$. in $\mathrm{NaCl}$-Lösung gegenüber dem Saponin, gegenüber $\mathrm{NaOH}$ und gegenüber den Serumhämolysinen resistenter als die alten.

Die ersten hierher gehörenden Versuche aus dem Jahre 1912²) hatten

1) Vgl. Anm. 2.

2) Handovsky, Arch. f. exp. Pathol. u. Pharmakol. 69, 412. 1912. Dort wurden zum ersten Male die Unterschiede der Giftresistenz von Rz.- und NaCl-Bl. systematisch untersucht; danach hat sich Ph. Eisenberg (Zentralbl. f. Bakteriol. usw. Abt. II, 69, 187. 1913) prinzipiell mit der Frage der Beeinflussung der durch verschiedene Stoffe hervorgerufenen Hämolyse durch Salze vom Standpunkt der Desinfektionslehre beschäftigt und prinzipiell das gleiche qualitative Verhalten beobachtet, daß die Resistenz der $\mathrm{Bl}$. in $\mathrm{NaCl}$ kleiner ist als in Rz. Schließlich hat Herr H. Rhode in unserem Institut gelegentlich einer Untersuchung über die hämolysierenden Eigenschaften verschiedener Salze von Alkaloiden auch die 
folgende GesetzmäBigkeit ergeben ${ }^{1}$ ): Die Verstärkung der hämolytischen Saponinwirkung durch $\mathrm{NaCl}$ (gegenüber Rz.) ist innerhalb gewisser Konzentrationsintervalle der NaCl- und der „Wirkungskonzentration“" des Saponins proportional. Es ließ sich für die damaligen Versuche, die mit Saponinum purissimum Merck ausgeführt wurden, folgende Formel aufstellen: $H=H_{0}^{\circ}+2500 c \cdot c_{\text {Sap }}$, wobei die Schwellenkonzentration $0,0048 \%$ Saponin betrug. $H$ bedeutet hierbei den Hämolysegrad $(\%)$ in Salzlösung, $H_{0}$ den in Rz.-Lösung, $c$ die Millimole Salz in $100 \mathrm{ccm}, c_{\text {Sap }}$ die Wirkungskonzentration des Saponins.

Als die Versuche jetzt wieder aufgenommen wurden (1921), ergaben sich für die Verstärkung der Saponin-Rz.-Bl.-Hämolyse andere Konstanten in der Formel, und zwar: $H=H_{0}+495 c \cdot c_{\text {Sap }}$, wobei die Schwellenkonzentration $0,0014 \%$ Saponin entspricht (vgl. Tab. IV).

Das zu diesen Versuchen verwendete Saponinpräparat, das ich im Institut vorfand, Saponinum purissimum albissimum Merck, wirkte also auf $\mathrm{NaCl}$ - und $\mathrm{Rz} .-\mathrm{Bl}$. stärker giftig ein; dementsprechend war die Giftverstärkung, da ja die Auflösung der Zellen der Giftwirkung ein Ende setzte, geringer. Die weiteren Versuche wurden alle mit demselben giftigeren Saponinpräparat gemacht.

\section{Versuche mit NaCl.}

Tabelle IV.

$H=H_{0}+495 c \cdot c_{\text {Sap }}$ (Schwellenwertkonzentration $0,0014 \%$ ).

\begin{tabular}{|c|c|c|c|c|c|c|c|c|c|c|}
\hline & \multirow{2}{*}{\multicolumn{2}{|c|}{$\begin{array}{c}0,006^{\circ} / 00 \text { Sap } \\
H=H_{0}+2,24 c \\
H_{0}=16 \\
H\end{array}$}} & \multirow{2}{*}{\multicolumn{2}{|c|}{$\begin{array}{c}0,008^{\circ} \%_{00} \mathrm{Sap} \\
\Rightarrow=H_{0}+3,24 e \\
H_{0}=21 \\
H\end{array}$}} & \multirow{2}{*}{\multicolumn{2}{|c|}{$\begin{array}{c}\begin{array}{c}0,04^{0} / 00 \\
H=H_{0}+4,24 c \\
H_{0}=26\end{array} \\
H\end{array}$}} & \multirow{2}{*}{\multicolumn{2}{|c|}{$\begin{array}{c}0,012^{\circ} \%_{00} \mathrm{Sap} \\
H=H_{0}+5,24 c \\
H_{0}=\mathbf{=}=\mathbf{c}\end{array}$}} & \multirow{2}{*}{\multicolumn{2}{|c|}{$\begin{array}{c}0,016^{0} \% \text { Sap } \\
H=H_{0}+7,24 c \\
H_{0}=40 \\
H\end{array}$}} \\
\hline & & & & & & & & & & \\
\hline & gef. & ber. & gef. & ber. & gef. & ber. & gef. & ber. & gef. & ber. \\
\hline 1,54 & 20 & 20 & 25 & 26 & 33 & 33 & 38 & 39 & 50 & 54 \\
\hline 3,08 & 23 & 23 & 30 & 31 & 45 & 39 & 55 & 48 & 70 & 69 \\
\hline 7,70 & 29 & 33 & 44 & 46 & 61 & 58 & 73 & 72 & - & - \\
\hline 12,32 & 42 & 44 & 72 & 71 & - & - & 一 & $\rightarrow$ & - & - \\
\hline
\end{tabular}

Diese Befunde entsprechen, wie schon erwähnt, sehr gut der Formel: $H=H_{0}+495 c \cdot c_{\text {sap }}$ (Schwellenkonzentration $0,0014 \%$ Saponin).

\section{Versuche mit NaJ.}

Diese Versuche entsprechen der Formel $H={ }_{0} H+250 c \cdot c_{\text {Sap }}$, wobei die Schwellenkonzentration $0,0048^{\circ} \%$ beträgt. Auffallend ist die geringe verstärkende Wirkung des $\mathrm{NaJ}$ gegenüber dem $\mathrm{NaCl}$ und

Resistenz der $\mathrm{B} 1$. gegenüber denselben in $\mathrm{Rz}$. und $\mathrm{NaCl}$ untersucht und gleichfalls gefunden, daß sie in ersterem wesentlich größer ist; da ich das gleiche für die $\mathrm{NaOH}$ und Serumhämolyse beobachtet habe, bilden diese Untersuchungen somit eine ergänzende Bestätigung des qualitativ scheinbar allgemein gültigen Befundes, da $B$ Bl. in Rz. resistenter sind als in Salzlösungen.

1) Dies. Arch. 190, 173. 1921. 
die ausgezeichnete Übereinstimmung der gefundenen und berechneten $H$-Werte (vgl. Tab. V).

T'abelle $V$.

$H=H_{0}+250 c \cdot c_{\text {Sap }}$ (Schwellenwertkonzentration 0,0048\%).

\begin{tabular}{|c|c|c|c|c|c|c|c|c|c|c|}
\hline \multirow{3}{*}{$c$} & \multirow{2}{*}{\multicolumn{2}{|c|}{$\begin{array}{c}0,006 \% \text { Sap } \\
\begin{array}{c}H=H_{0}+0,32 c \\
H_{0}=16\end{array} \\
H\end{array}$}} & \multirow{2}{*}{\multicolumn{2}{|c|}{$\begin{array}{c}0,008^{\circ} / \text { Sap } \\
H=H_{0}+0,82 c \\
H_{0}=21 \\
H\end{array}$}} & \multirow{2}{*}{\multicolumn{2}{|c|}{$\begin{array}{c}0,01^{\circ} \% \text { Sap } \\
H=H_{0}+1,30 c \\
H_{0}=26\end{array}$}} & \multirow{2}{*}{\multicolumn{2}{|c|}{$\begin{array}{c}0,012^{\circ} / \mathrm{Sap} \\
H=H_{0}+1 \cdot 70 c \\
H_{0}=\mathbf{B 1} \\
H\end{array}$}} & \multirow{2}{*}{\multicolumn{2}{|c|}{$\begin{array}{c}0,016^{\circ} \text { Sap } \\
H=H_{0}+2,60 c \\
H_{0}=40\end{array}$}} \\
\hline & & & & & & & & & & \\
\hline & gef. & ber. & gef. & ber. & gef. & ber. & gef. & ber. & gef. & ber. \\
\hline 1,5 & 16 & 16 & 20 & & 2 & 2 & 3 & 34 & 39 & 40 \\
\hline 3,0 & 16 & $1 ?$ & 23 & 2 & 30 & 3 & 3 & 36 & 48 & 48 \\
\hline 7,7 & 18 & 18 & 27 & 2 & 34 & 36 & 42 & 44 & 58 & 60 \\
\hline 12,3 & 21 & 20 & 30 & 31 & 39 & 42 & 51 & 52 & 72 & 74 \\
\hline 15,40 & 22 & 21 & 34 & 34 & 46 & 46 & 57 & 57 & 82 & 80 \\
\hline
\end{tabular}

Viel schwieriger ist die Aufstellung einer entsprechenden Formel für die Wirkung von $\mathrm{Na}_{2} \mathrm{SO}_{4}$.

\section{Versuche mit $\mathrm{Na}_{2} \mathrm{SO}_{4}$.}

Tabelle VI.

\begin{tabular}{|c|c|c|c|c|c|c|c|}
\hline$c$ & $\begin{array}{c}0,002 \\
\% \text { \%ap }\end{array}$ & $\begin{array}{c}0,004 \\
\% \text { \%o Sap }\end{array}$ & $\begin{array}{c}0,006 \\
\% \text { Sap }\end{array}$ & $\begin{array}{c}0,008 \\
\% \text { Sap } \\
H \text { gefunden }\end{array}$ & $\begin{array}{c}0,010 \\
\% \text { Sap }\end{array}$ & $\begin{array}{c}0,012 \\
\% \text { Sap }\end{array}$ & $\begin{array}{c}0,016 \\
\% \text { Sap }\end{array}$ \\
\hline 3,08 & - & 15 & 26 & 32 & 39 & 45 & 58 \\
\hline 7,70 & - & 17 & 30 & 47 & 55 & 72 & 88 \\
\hline 12,32 & - & 25 & 40 & 55 & 77 & 88 & 96 \\
\hline 15,40 & 18 & 36 & 51 & 70 & 83 & 96 & 100 \\
\hline
\end{tabular}

Aus diesen Versuchen konnte ich eine einheitliche allgemeingültige Formel nicht berechnen. Bei den gegen $0,01-0,016 \%$ Saponin eben empfindlichen $\mathrm{Bl}$. entspricht die Verstärkung der Giftwirkung sehr gut der Formel $H=H_{0}+710 c \cdot c_{\text {Sap }}$, wobei sich als Schwellenkonzentration $0,0048 \%$ ergibt. Für die älteren Bl., die bereits durch $0,004-0,008 \%$ Saponin vergiftet werden, stimmt diese Formel gar nicht; hingegen stimmen die $H$-Werte in diesen Fällen ganz gut mit den nach einer Formel $H=H_{0}+345 c \cdot c_{\text {Sap }}$ berechneten überein, wenn als Schwellenkonzentration Null gesetzt wird; das würde bedeuten, daß es $\mathrm{Bl}$. gibt, die in $\mathrm{Na}_{2} \mathrm{SO}_{4}$-haltigen Medien schon durch die geringsten Spuren Saponin gelöst werden. Daß wir bei den $\mathrm{Na}_{2} \mathrm{SO}_{4}$-Versuchen zu keiner einheitlichen Formel kamen, wie beim $\mathrm{NaCl}$ oder $\mathrm{NaJ}$, dürfte seinen Grund darin haben, daß Bl., die in einer NaCl-Lösung noch zu den mittelalten gehören, in $\mathrm{Na}_{2} \mathrm{SO}_{4}$-haltigen Lösungen bereits gealtert sind; die Alterserscheinungen können daher in verschiedenen Medien in verschiedenen Lebensaltern eintreten.

Noch komplizierter sind die Verhältnisse beim NaSCN. 


\section{Versuche mit NaSCN.}

Tabelle VII.

\begin{tabular}{|c|c|c|c|c|c|c|}
\hline \multirow[t]{2}{*}{$c$} & $\begin{array}{c}0,004 \\
\% \% 0 \text { Sap }\end{array}$ & $\begin{array}{c}0,006 \\
\% \text { \%o Sap }\end{array}$ & $\begin{array}{c}0,000 \\
\% \\
\% \text { \% } 8 \mathrm{ap}\end{array}$ & $\begin{array}{c}0,010 \\
\% \text { \%o Sap }\end{array}$ & $\begin{array}{c}0,012 \\
\% \% 0 \text { Sap }\end{array}$ & $\begin{array}{c}0,016 \\
\% \% 0 \text { Sap }\end{array}$ \\
\hline & \multicolumn{6}{|c|}{$H$ gefunden } \\
\hline 3,08 & - & 19 & 26 & 32 & 39 & 52 \\
\hline 7,70 & - & 19 & 33 & 45 & 58 & 78 \\
\hline 12,32 & - & 34 & 65 & 81 & 90 & 98 \\
\hline 15,40 & 15 & 48 & 80 & 96 & 100 & $\ldots$ \\
\hline
\end{tabular}

Auch beim NaSCN ist eine giftverstärkende Wirkung vorhanden, die mit zunehmender Salz- und Saponinkonzentration zunimmt; aber diese Zunahme ist der Konzentrationszunahme nicht proportional, wie wir dies bei den bisher besprochenen Salzen gesehen haben, sondern sie ist stärker, als es der Salzkonzentration entspricht; es muß also wohl das SCN.Ion nicht nur eine giftverstärkende, sondern auch selbst eine Giftwirkung im Sinne einer Hämolyse ausüben. Eine entsprechende Sonderstellung des SCN-Ions beobachtete auch I. Runnström ${ }^{\mathbf{1}}$ ) bei Untersuchung der durch Salze bewirkten Stabilisierung von Bl. gegen die Sedimentation.

Was nun die Wirkung von Salzen mit anderen Kationen anlangt, paßt sich das $\mathrm{LiCl}$ sehr schön dem $\mathrm{NaCl}$ an.

\section{Versuche mit LiCl.}

Tabelle VIII.

\begin{tabular}{|c|c|c|c|c|c|c|c|c|}
\hline \multirow[t]{3}{*}{ c } & \multirow{2}{*}{\multicolumn{2}{|c|}{$\begin{array}{c}0,006 \% \text { Sap } \\
\begin{array}{c}H=H_{0}+1,45 c \\
H_{0}\end{array}=16^{2} \\
H\end{array}$}} & \multirow{2}{*}{\multicolumn{2}{|c|}{$\begin{array}{c}\begin{array}{c}0,008 \% \\
H=H_{0}+4,05 \\
H_{0}=21\end{array} \\
H\end{array}$}} & \multirow{2}{*}{\multicolumn{2}{|c|}{$\begin{array}{c}0,01 \% \text { Sap } \\
H=H_{0}+6,65 c \\
H_{0}=26\end{array}$}} & \multirow{2}{*}{\multicolumn{2}{|c|}{$\begin{array}{c}0,012 \% \text { Sap } \\
H=H_{0}+9,25 c \\
H_{0}=31\end{array}$}} \\
\hline & & & & & & & & \\
\hline & gef. & ber. & gef. & ber. & gef. & ber. & gef. & ber. \\
\hline 3,08 & 16 & 20 & 29 & 33 & 46 & 46 & 59 & 59 \\
\hline 7,70 & 29 & 27 & 51 & 51 & 74 & 77 & 85 & $\ldots$ \\
\hline 12,32 & 43 & 34 & 73 & 71 & 85 & $\ldots$ & 97 & $\ldots$ \\
\hline 15,40 & 89 & 88 & 96 & - & 100 & - & - & - \\
\hline
\end{tabular}

Zur Charakterisierung der Wirkung des $\mathrm{LiCl}$ genügt die Formel $H=H_{2}+1260 c \cdot c_{\text {Sap }}$ ganz gut, wobei sich als Schwellenkonzentration $0,0048 \%$ errechnet; die giftverstärkende Wirkung des $\mathrm{LiCl}$ ist größer als die des $\mathrm{NaCl}$, was sehr gut mit anderen biologischen Wirkungen des Li-Ions übereinstimmt.

Viel komplizierter gestalten sich die Beziehungen bei Salzen, die selbst hämolytisch wirken. Hierher gehören die $\mathrm{Mg}$ - und Ca-Salze, mit denen folgende Versuche gemacht wurden.

1) Biochem. Zeitschr. 123, 1. 1921. 


\section{6.-13. Versuche mit Mg- und Ca-Salzen.}

6. $\mathrm{MgSO}_{4}$ und Rz.-Bl.

Tabelle IX.

\begin{tabular}{|c|c|c|c|c|c|c|c|}
\hline$e_{\downarrow}$ & $\begin{array}{l}c_{\mathrm{Sap}} \rightarrow \\
c_{\mathrm{Rz}} \downarrow\end{array}$ & 0,000 & $0,004 \%$ & $\begin{array}{l}0,006 \% \\
\tilde{H}_{0}=16\end{array}$ & $\begin{array}{c}0,010 \% \\
H_{0}=26\end{array}$ & $\begin{array}{c}0,012 \% \\
H_{0}=31\end{array}$ & Volum \\
\hline $0,0154 \mathrm{n}$ & $7,02 \%$ & 10 & 14 & 51 & 80 & 90 & 0,0060 \\
\hline $0,0308 n$ & $6,24 \%$ & 16 & 28 & 56 & 95 & 95 & 0,0058 \\
\hline $0,0770 \mathrm{n}$ & $3,90 \%$ & 21 & 52 & 63 & - & 96 & 0,0060 \\
\hline $0,1232 \mathrm{n}$ & $1,56 \%$ & 93 & 80 & 86 & 78 & 96 & 0,0077 \\
\hline $0,1540 \mathrm{n}$ & 0 & 100 & 100 & 100 & 100 & 100 & - \\
\hline
\end{tabular}

7. $\mathrm{Mg}(\mathrm{SCN})_{2}$ und Rz.-Bl.

Tabelle $X$.

\begin{tabular}{c||c|c|c|c|c|c|c}
\hline $\begin{array}{c}c_{\mathrm{S} \text { sap }} \rightarrow \\
{ }_{\downarrow}\end{array}$ & 0,000 & $0,004 \%$ & $0,005 \%$ & $0,010 \%$ & $0,012 \%$ & $0,016 \%$ & $0,025 \%$ \\
\hline $0,0308 \mathrm{n}$ & 28 & $\mathbf{1 6}$ & 37 & 50 & 65 & 78 & 96 \\
$0,0770 \mathrm{n}$ & $\mathbf{3 3}$ & 20 & 42 & 55 & 73 & 94 & 96 \\
$0,1232 \mathrm{n}$ & $\mathbf{3 5}$ & 28 & 53 & 65 & 82 & - & - \\
$0,1540 \mathrm{n}$ & 96 & 88 & 80 & 80 & 80 & 85 & 90
\end{tabular}

8. $\mathrm{MgSO}_{4}$ und $\mathrm{NaCl}-\mathrm{Bl}$.

Tabelle XI.

\begin{tabular}{l||c|r|r|r|r|r|r}
\hline \multicolumn{1}{c|}{$c_{\downarrow}$} & $\begin{array}{c}c_{\mathrm{sap} \rightarrow} \rightarrow \\
c_{\mathrm{NaCl} \downarrow}\end{array}$ & 0 & $0,004 \%$ & $0,006 \%$ & $0,010 \%$ & $0,012 \%$ & Volum *) \\
\hline $0 \mathrm{n}$ & $0,154 \mathrm{n}$ & 0 & 50 & 91 & 100 & - & 0,0072 \\
0,0154 & 0,1386 & 25 & 51 & 64 & 84 & 96 & 0,0063 \\
0,0308 & 0,1232 & 9 & 38 & 55 & 76 & 80 & 0,0074 \\
0,0770 & 0,0770 & 61 & 64 & 75 & 78 & 81 & 0,0055 \\
0,1232 & 0,0308 & 100 & & & & & \\
0,1540 & 0 & & & & & &
\end{tabular}

9. $\mathrm{CaBr}_{2}$ und $\mathrm{Rz},-\mathrm{Bl}$.

Tabelle XII.

\begin{tabular}{c||c|c|c|c|c|c|c}
\hline \hline$c_{\downarrow}$ & $\begin{array}{l}c_{\text {sap }} \rightarrow \\
c_{\mathrm{Rg}}\end{array}$ & 0 & $0,001 \%$ & $0,006 \%$ & $0,008 \%$ & $0,010 \%$ & Voium*) \\
\hline \hline $0,0154 \mathrm{n}$ & $7,02 \%$ & 0 & 21 & 58 & 79 & 96 & 0,0046 \\
$0,0308 \mathrm{n}$ & $6,24 \% \%$ & 0 & 40 & 73 & 83 & 96 & \\
$0,0770 \mathrm{n}$ & $3,90 \%$ & 14 & 54 & 84 & 96 & 100 & 0,0062 \\
$0,1232 \mathrm{n}$ & $1,56 \%$ & 21 & 58 & 93 & 96 & 100 & \\
$0,1540 \mathrm{n}$ & $0 \%$ & 24 & 68 & 88 & 100 & 100 & 0,0062
\end{tabular}


10. Ca(SCN $)_{2}$ und Rz.-Bl.

\begin{tabular}{l||c|c|c|c|c|c|c}
\multicolumn{8}{c}{ Tabelle XIII. } \\
\hline$c_{\downarrow}$ & $\begin{array}{l}c_{\text {sap } \rightarrow} \\
c_{\mathrm{Rz} \downarrow}\end{array}$ & 0 & $0,004 \%$ & $0,006 \%$ & $0,008 \%$ & $0,010 \%$ & Volum *) \\
\hline 0,0308 & $6,24 \%$ & 28 & 44 & 65 & 88 & 96 & 0,0056 \\
0,0770 & $3,90 \%$ & 34 & 41 & 67 & 90 & 96 & 0,0043 \\
0,1232 & $1,56 \%$ & 42 & 68 & 85 & 90 & 96 & \\
0,1540 & $0 \%$ & 85 & 87 & 91 & 95 & 96 &
\end{tabular}

11. $\mathrm{CaCl}_{2}$ und Rz.-BI.

Tabelle XIV.

\begin{tabular}{c||c|c|c|c|c|c}
\hline \hline$c_{\downarrow}$ & $\begin{array}{c}c_{\mathrm{sap} \rightarrow} \\
c_{\mathrm{Rz} \downarrow}\end{array}$ & 0 & $0,004 \%$ & $0,008 \%$ & $0,008 \%$ & $0,010 \%$ \\
\hline 0,0154 & $7,02 \%$ & 0 & 61 & 80 & 92 & 98 \\
0,0308 & $6,24 \%$ & 0 & 48 & 70 & 82 & 95 \\
0,0770 & $3,90 \%$ & 24 & 25 & 68 & 78 & 90 \\
0,1232 & $1,56 \%$ & 60 & 62 & 86 & 98 & 100 \\
0,1540 & $0 \%$ & 90 & 98 & 100 & - & -
\end{tabular}

12. $\mathrm{CaBr}_{2}$ und $\mathrm{NaCl}-\mathrm{Bl}$.

Täbelle XV.

\begin{tabular}{|c|c|c|c|c|c|c|c|}
\hline$c_{\downarrow}$ & $\begin{array}{l}c_{\mathrm{Sap}} \rightarrow \\
c_{\mathrm{NaCl}} \downarrow\end{array}$ & 0 & $0,004 \%$ & $0,006 \%$ & $0,008 \%$ & $0,010 \%$ & Volum $*$ ) \\
\hline $0 \mathbf{n}$ & $0,154 \mathrm{n}$ & 0 & 52 & 91 & 95 & 100 & 0,0088 \\
\hline 0,0154 & 0,1386 & 0 & 14 & 65 & 80 & 96 & \\
\hline 0,0808 & 0,1232 & 40 & 73 & 91 & 96 & 100 & 0,0052 \\
\hline 0,0770 & 0,0770 & 0 & 51 & 84 & 96 & 100 & 0,0088 \\
\hline 0,1232 & 0,0308 & 0 & 51 & 78 & 一 & 100 & \\
\hline 0,1540 & 0 & 20 & 54 & 72 & 100 & 100 & 0,0078 \\
\hline
\end{tabular}

14. Kombinierte $\mathrm{MgSO}_{4}$ und $\mathrm{CaBr}_{2}$-Wirkung auf Rz.-Bl.

Tabelle XVI.

\begin{tabular}{c|c|c|c|c|c|c}
\hline $\mathrm{MgSO}_{4}$ & $\mathrm{CaBr}_{2}$ & $\mathrm{Rz}$ & NaCl & Sap & H & Volum \\
\hline \hline $0,077 \mathrm{n}$ & - & $3,92 \%$ & - & 0 & 28 & 0,0083 \\
& & & & 0,004 & 35 & \\
& & & & 0,006 & 50 & \\
$0,046 \mathrm{n}$ & \multirow{3}{*}{0,031} & \multirow{3}{*}{$3,92 \%$} & - & 0,010 & 62 & \\
& & & & 0,004 & 35 & \\
& & & & 0,006 & 48 & \\
& & & & 0,010 & 81 &
\end{tabular}


Tabelle XVI (Fortsetzung).

\begin{tabular}{c|c|c|c|c|c|c}
\hline \hline $\mathrm{MgSO}_{4}$ & $\mathrm{CaBr}_{2}$ & $\mathrm{Rz}$ & $\mathrm{NaCl}$ & $\mathrm{Sap}$ & $\mathrm{H}$ & Volum \\
\hline \multirow{2}{*}{0,015} & 0,062 & $3,92 \%$ & - & 0 & 10 & - \\
& & & & 0,004 & $\mathbf{4 4}$ & \\
& & & & 0,006 & 81 & \\
& \multirow{3}{*}{$0,077 \mathrm{n}$} & \multirow{3}{*}{$3,92 \%$} & - & 0,010 & 92 & \\
& & & & 0,004 & 51 & \\
& & & & 0,006 & 81 & \\
& & & & 0,010 & 96 &
\end{tabular}

Aus der Fülle von Einzelheiten, die in diesen Versuchen beobachtet werden konnten, seien folgende hervorgehoben:

1. Alle Mg-Salze verstärken die Wirkung des Saponins gegenüber Rz.-Bl., und zwar $\mathrm{MgSO}_{4}$ mehr als $\mathrm{Mg}(\mathrm{SCN})_{2}$.

2. Es besteht eine ausgesprochene antagonistische Wirkung von $\mathrm{Rz}$. und $\mathrm{Mg}$ auf die Saponinempfindlichkeit der Bl.; schon Spuren von Rz. hemmen die Mg-Hämolyse beträchtlich, während die Ca Hämolyse entsprechend dem Ca-Gehalt zunimmt, recht unabhängig vom Rz.-Gøhalt. Diese Annahme wird auch durch folgenden instruktiven Versuch gestützt: In einer 0,154 n-MgSO $4^{-}$sowohl als auch in einer $0,154 \mathrm{n}-\mathrm{CaCl}_{2}$-Lösung werden die Bl. in kurzer Zeit schon bei Zimmertemperatur komplett gelöst; werden zu beiden einmal $\mathrm{Rz}$ das andere $\mathrm{Mal} \mathrm{NaCl}$ in Mengen, hinzugefügt, so daß alle Mischungen doppelt isotonisch sind, dann wird die $\mathrm{MgSO}_{4}$-Hämolyse durch $\mathrm{Rz}$, nicht aber durch $\mathrm{NaCl}$-Überschuß gehemmt, die $\mathrm{CaCl}_{2}-\mathrm{Hämolyse}$ weder durch $\mathrm{Rz}$, noch durch $\mathrm{NaCl}$. Der Antagonismus erstreckt sich insbesondere auf die mittelalten und jungen Bl., denn obwohl z. B. in einer $0,154 \mathrm{n}-\mathrm{Mg}(\mathrm{SCN})_{2}$-Lösung $96 \%$ Bl. gelöst werden, sind in derselben Lösung $+0,01 \%$ Saponin nur $80 \% \mathrm{Bl}$. gelöst (Tab. X). Die Mg-Salze schützen also besonders die jungen Bl. vor der Saponinvergiftung.

3. Wird ein Teil des $\mathrm{NaCl}$ durch die gleiche Anzahl Mole $\mathrm{MgSO}_{4}$ ersetzt, dann hängt die hämolytische Wirkung vom Verhältnis $\frac{\mathrm{MgSO}_{4}}{\mathrm{NaCl}}$ ab; sie hat, wenn $\frac{\mathrm{MgSO}_{4}}{\mathrm{NaCl}}=0,11$ ein Maximum, nimmt dann bis $\frac{\mathrm{MgSO}_{4}}{\mathrm{NaCl}}$ $=0,25 \mathrm{ab}$ und steigt von da wieder an (Tab. XI).

4. Wird ein Teil des $\mathrm{NaCl}$ durch die gleiche Anzahl Mole $\mathrm{MgSO}_{4}$ ersetzt, dann wird die Giftempfindlichkeit der jüngeren $\mathrm{Bl}$. vermindert, die der älteren gesteigert; die verstärkende Wirkung nimmt mit zunehmendem Mg-Gehalt ab.

5. Die Ca-Salze üben auch stets eine verstärkende Wirkung auf die Saponinvergiftung der Rz.-Bl. aus, die von der Salzkonzentration 
ziemlich unabhängig ist. Beim $\mathrm{CaCl}_{2}$ geht sie durch ein Maximum. Von den untersuchten Konzentrationen wirkt die am stärksten lytisch wirkende Konzentration am schwächsten verstärkend (Tab. XIV).

6. Ersatz von $\mathrm{NaCl}$ durch kleine Mengen $\mathrm{CaBr}_{2}$ vermindert die Giftempfindlichkeit der Bl. beträchtlich; in höheren Konzentrationen, also bei zunehmendem Verhältnis $\frac{\mathrm{Ca}}{\mathrm{Na}}$, wird die Giftempfindlichkeit der alten Zellen beträchtlich gesteigert, die der jungen, wenn auch in geringem Ausmaß, vermindert (Tab. XV).

7. Der bekannte biologische und allgemein kolloidchemische Antagonismus von ein- und zweiwertigen Ionen gilt also auch für die Beeinflussung der Giftempfindlichkeit von Bl. gegenüber Saponin. Ein Antagonismus von $\mathrm{Ca}$ und $\mathrm{Mg}$ konnte nicht beobachtet werden, höchstens in bezug auf die jungen Zellen, deren Giftempfindlichkeit durch Mg-Salze beträchtlich herabgesetzt wird, nicht aber durch Ca-Salze.

Wenn wir jetzt daran gehen, die obigen Versuche durch kolloidchemische Überlegungen auf eine einheitliche Basis zu stellen, müssen wir zunächst noch unsere Erfahrungen über den Zusammenhang von Giftempfindlichkeit und Volumen der Bl. sammeln.

Die Volumina der Rz.-Bl. sind bei den verschiedenen Blutproben natürlich nicht gleich, ebensowenig die der $\mathrm{NaCl}-\mathrm{Bl}$,, wohl aber ist das Volumen der Bl. derselben Entnahme in 7,8 proz. Rz. stets um ca. 20\% geringer als in $0,154 \mathrm{n}-\mathrm{NaCl}-\mathrm{Lösung}$ (vgl. Tab. XVII).

Tabelle XVII.

Hier und im folgenden ist unter Volumen stets das Volumen von 109 Millionen Bl., und zwar in Kubikzentimeter gemeint. 109 Millionen sind in $1 \mathrm{ccm}$ einer 2,5 proz. Kaninchenblutsuspension durchschnittlich vorhanden.

Volumina der Blutkörperchen in $1 \mathrm{ccm} 2,5$ proz. Blut aufgeschwemmt in

\begin{tabular}{c|c}
\hline $7,8 \%$ Rohrzucker & $0,9 \%$ NaCl \\
\hline \hline 0,0045 & $\overline{00060}$ \\
0,0046 & 0,0070 \\
0,0058 & 0,0072 \\
0,0060 & 0,0073 \\
0,0060 & 0,0074 \\
0,0060 & 0,0076 \\
- & 0,0076 \\
- & 0,0076 \\
0,0072 & 0,0086 \\
0,0072 & 0,0091 \\
\hline Mittel : 0,0059 & 0,0073
\end{tabular}

Eine Ursache für das verschiedene Volumen der Bl. verschiedener Kaninchen im gleichen Milieu konnte ich nicht auffinden. Nachdem ich 
die Arbeit von $\mathrm{Ege}^{1}$ ) gelesen hatte, habe ich Kontrollen der Volumbestimmung von $\mathrm{NaCl}$ und auch Rz.-Bl. gemacht, indem ich das Volumen zu Beginn des Versuches, etwa $3 / 4$ Stunden nach dem Vermischen der Bl. mit ihrer Suspensionsflüssigkeit, nach Herausnehmen aus dem Brutschrank, also etwa $21 / 4$ Stunden später und nach etwa 10 stündigem Verweilen im Eisschrank bestimmte. Ich konnte keinerlei Unterschiede in der Größe des BI.-Volumens zu diesen 3 verschiedenen Zeitpunkten finden, was ja schließlich den Befunden Eges nicht direkt widerspricht. Die Volumbestimmung geschah stets in selbst nachtarierten Hamburgerschen Chronohämatokriten mit 0,02 bzw. $0,04 \mathrm{ccm}$ Capillarinhalt; ich mußte stets $1 \frac{1}{2}-1^{3} / 4$ Stunde bis zur Volumkonstanz zentrifugieren.

Eine darauf gerichtete Durchsicht der oben mitgeteilten Tabellen lehrt allenthalben, daß ein Zusammenhang zwischen Volumen und Giftempfindlichkeit der Bl. nicht besteht. Besonders eklatant erscheint ein Versuch, in dem Bl. derselben Entnahme unter 2 verschiedenen Bedingungen ein Volumen von $0,0072 \mathrm{ccm}$ hatten, aber durch $0,008 \%$ Saponin im einen Fall zu 30\%, im anderen zu 96\% hämolysiert wurden. 'Das gleiche beweist der Einfluß der Konzentration des Rz. auf Giftempfindlichkeit und Volumen der Bl. (vgl. Tab. XVIII).

Tabelle XVIII.

\begin{tabular}{c|c|c|c|c}
\hline $\begin{array}{c}c_{\mathrm{Rz}} \\
\%\end{array}$ & \multicolumn{2}{|c|}{ Volum } & \multicolumn{2}{|c}{$H$ (Versuch 43) } \\
\hline 5,85 & Versuch 26 & Versuch 43 & $0,012 \%$ Sap & $0,025 \%$ Sap \\
\hline 7,8 & 0,0049 & 0,0059 & 32 & 84 \\
15,6 & 0,0047 & 0,0056 & 34 & 62 \\
& 0,0037 & 0,0087 & 32 & 62
\end{tabular}

Trotz des mit zunehmender Rz.-Konzentration kontinuierlich abnehmenden Volums, eine Erscheinung, die inzwischen von T. Take $i^{2}$ ) im Hamburgerschen Institut systematisch untersucht wurde, ist die Giftempfindlichkeit in hypo- bis hypertonischen Lösungen gleich, in hypotonischen erscheint die Resistenz der jungen Bl. vermindert.

In diesem Zusammenhang muß noch ein Versuch erwähnt werden: Es ist, wie ich mich wiederholt überzeugen konnte, für Volumen und Saponinempfindlichkeit der Bl. gleichgültig, ob diese vor dem Aufschwemmen in $\mathrm{Rz}$. in $\mathrm{NaCl}$ oder in $\mathrm{Rz}$. serumfrei gewaschen wurden. Nicht gleichgültig ist dies jedoch für $\mathrm{Bl}$., die in $\mathrm{NaCl}$ aufgeschwemmt werden sollen. Schon Gürber ${ }^{3}$ ) hatte beobachtet, daß in Rz. gewaschene $\mathrm{Bl}$. gegen $\mathrm{CO}_{2}$ ungemein empfindlich sind. Ich konnte zweimal beobachten, daß in Rz. bis zur Cl-Freiheit der Waschflüssigkeit gewaschene

1) Biochem. Zeitschr. 115, 109. 1920 und ebenda 115, 175.1921.

2) Biochem. Zeitschr. 123, 126. 1921.

3) Habilitationsschrift. Würzburg 1904.

Pflügers Archiv f. d. ges. Physiol, Bd. 195. 
264 H. Handovsky: Quantitative Beiträge zur Frage des Zusammenwirkens

Bl. gegenüber $0,154 \mathrm{n}-\mathrm{NaCl}$ sehr empfindlich geworden sind; das $\mathrm{NaCl}$ wirkt dann so wie sonst Salze mit zweiwertigen Kationen (vgl. Tab. XIX).

Tabelle XIX.

\begin{tabular}{|c|c|c|c|c|c|}
\hline \multirow{2}{*}{$\frac{\mathrm{Rz}_{\mathrm{z}}{ }^{2}}{\mathrm{Na}}$} & \multirow{2}{*}{$\begin{array}{c}c_{\mathrm{Sap}} \\
\% / 00\end{array}$} & \multicolumn{2}{|c|}{ Rohrzucker-Waschung ${ }^{1}$ ) } & \multicolumn{2}{|c|}{ NaCl-Waschung ${ }^{2}$ ) } \\
\hline & & $\begin{array}{l}H \\
\% \\
\end{array}$ & Vol. & $\begin{array}{l}H \\
\%\end{array}$ & Vol. \\
\hline$\frac{5}{1}$ & $\begin{array}{l}0 \\
0,004 \\
0,006 \\
0,010\end{array}$ & $\begin{array}{l}0 \\
0 \\
16 \\
21\end{array}$ & $\begin{array}{l}0,0058 \\
0,0050\end{array}$ & $\begin{array}{l}0 \\
0 \\
16 \\
21\end{array}$ & 0,0058 \\
\hline$\frac{4}{1}$ & $\begin{array}{l}0 \\
0,008\end{array}$ & $\begin{array}{l}10 \\
26\end{array}$ & & $\begin{array}{l}0 \\
25\end{array}$ & \\
\hline$\frac{2,5}{2,5}$ & $\begin{array}{l}0 \\
0,008\end{array}$ & $\begin{array}{l}14 \\
30\end{array}$ & 0,0072 & $\begin{array}{l}0 \\
32\end{array}$ & 0,0069 \\
\hline$\frac{1}{4}$ & $\begin{array}{l}0 \\
0,008\end{array}$ & $\begin{array}{l}36 \\
62\end{array}$ & & $\begin{array}{l}0 \\
65\end{array}$ & \\
\hline$-\frac{0}{5}$ & $\begin{array}{l}0 \\
0,004 \\
0,008\end{array}$ & $\begin{array}{l}96 \\
96 \\
100\end{array}$ & & $\begin{array}{l}0 \\
56 \\
96\end{array}$ & 0,0072 \\
\hline
\end{tabular}

Interessanterweise bleibt die Saponmempfindlichkeit die gleiche, ob die $\mathrm{NaCl}$-Bl. in $\mathrm{Rz}$. oder in $\mathrm{NaCl}$ gewaschen wurden. Die Ursachen der Hämolyse durch Salze und die der giftverstärkenden Wirkung der Salze sind also verschieden. Die hämolysierende Wirkung von Ionen beruht, wie Stewart $^{3}$ ) und neuerdings besonders Haffner ${ }^{4}$ ) nachgewiesen haben, auf Fällung oder Quellung von Hämoglobin oder Stroma, die verstärkende Wirkung jedoch, wie ich wahrscheinlich gemacht zu haben glaube ${ }^{5}$ ), auf einer Vergrößerung der freien Oberfläche, als Angriffspunkt des oberflächenaktiven Giftes $\left.^{6}\right)$. Wie können wir uns diese Oberflächenvergrößerung vorstellen? Da müssen wir uns zunächst der Frage zuwenden, in welchem kolloidchemischen Zustand sich die Rz.-Bl. befinden.

Die Rz.-Bl. haben stets ein ca. 20\% geringeres Volumen als NaCl-Bl. Um eine Entquellung im gewöhnlichen Sinn kann es sich hier schwer handeln; denn 1. ist von einer entquellenden Wirkung von Rz.

1) Blut derselben Entnahme.

2) $\frac{\mathrm{Rz}}{\mathrm{Na}}$ bedeutet das Verhältnis von Kubikzentimetern isotonischer Rohrzuckerzu isotonischer $\mathrm{NaCl}-\mathrm{Lösung}$.

3) Americ. Journ. of physiol. 11, 374. 1904.

4) Verhandl. d. dtsch. pharmakol. Ges. 1921, S. XXIX.

$\left.{ }^{5}\right)$ Pflügers Arch. f. d. ges. Physiol. 190, 173. 1921.

6) Utber die Unterschiede dieser verschiedenen Ursachen vgl. des Verf. Leitfarlen der Kolloidchemie f. Biol. u. Med. Dresden 1922, S. 185. 
nichts bekannt, 2. wäre eine Entquellung reversibel, während die Rz.-Bl. eine nachherige Aufschwemmung in $0,154 \mathrm{n}-\mathrm{NaCl}$ nicht vertragen. Wir stellen uns vielmehr vor, $d a \beta$ die $R z .-B l$. mehr gelatiniert sind als die $\mathrm{NaCl}-\mathrm{Bl}$; d die Volumabnahme dürfte so vor sich gehen, daß beim Gelatinieren oder erst beim Zentrifugieren - das wird sich experimentell schwer entscheiden lassen - der Rz.-Bl. Wasser ausgepreßt wird. Daß die Volumabnahme der Rz.-Bl. mit zunehmender Rz.-Konzentration fortschreitend zunimmt, spricht nicht gegen die Auffassung einer Gelatinierung; dafür gibt es vielmehr in der Kolloidchemie viele Beispiele ${ }^{\mathfrak{1}}$.

Die Gelatinierung ist stets mit einer Dispersitätsgradverminderung verbunden ${ }^{2}$ ). Die Salze wirken dieser Gelatinierung entgegen, erhöhen den Dispersitätsgrad und vergrößern die Angriffsfläche des Giftes. Für den mehr gelatinierten Zustand der Rz.-Bl. spricht auch der Antagonismus des Rz. gegenüber den Mg-Salzen (vgl. oben S. 259); denn die Mg-Salze sind als Verflüssiger des Protoplasmas bekannt ${ }^{3}$ ). Auch der oben angeführte (Tab. X) eigentümliche Befund, daß hämolysierende $\mathrm{Mg}(\mathrm{SCN})_{2}$-Konzentrationen durch geringe Saponinkonzentrationen in ihrer Wirkung antagonistisch beeinflußt werden, läßt sich in unserem Sinne deuten, da wir nach Untersuchungen von Knaffl-Lenz $z^{4}$ ) annehmen können, daß oberflächenaktive Stoffe in niedrigen Konzentrationen eine Verfestigung des Protoplasmas bedingen.

Wir kommen also zu folgender Vorstellung über die Ursachen der oben beschriebenen Phänomene: Der Rz. bewirkt innerhalb der wohl ständig hin und her pendelnden Sol-Gelumwandlung des Protoplasmas einen mehr gelatinierenden Zustand, dieser ist mit einer Verminderung des Dispersitätsgrades verbunden, die die Bl. Jür das oberflächenaktive Gift weniger empfindlich macht. Salze beeinflussen diesen Zustand in der entgegengesetzten Richtung; sie machen daher die Rz.-Bl. wieder giftempfindlicher, und zwar, was zugleich als Wahrscheinlichkeitsbeweis für diese Annahme angeführt sei, wenn sie selbst keine hämolytischen Wir. kungen ausüben, nach den für die Adsorptionsverstärkung geltenden Gesetzmäßigkeiten. Dabei ist es für diese Probleme natürlich belanglos, ob der Zustand der Rz. — oder der der $\mathrm{NaCl}$ - Bl. dem der Bl. im strömenden Blut näher kommt.

1) Vgl. Leitfaden, S. 173.

2) Vgl. Leitfaden, S. $163 \mathrm{ff}$.

3) Vgl. z. B. Hannsteen-Cranner, Jahrb. f. wiss. Botan. 53, 536. 1914.

4) Pflügers Arch. f. d. ges. Physiol. 171, 54. 1918. 